\title{
Adipogenic Differentiation of Canine Hair Follicle Stem Cells (cHFSCs)
}

\begin{abstract}
Background: Homeostasis in adult body is maintained by stem cells which is a powerful reservoir harbored in the body which regenerate tissue and in response to disease and injury.Hair follicle is a dynamic mini organ supporting important biological functions of the body in maintaining homeostasis and skin tissue self-renewal. Hair follicle protects the body from cold and potential injuries. To know the pluripotency nature of hair follicle stem cells, a study was conducted on canine HFSCs (cHFSCs).

Methods: In-vitro culture of cHFSCs was done by William's E medium with several growth factors and passaged. Stemness of the cultured cells was assessed by immunostaining of cells with Anti-cytokeratin 15 (CK15) marker. Viability of cells was checked by acridine orange staining and passage 2 cells were induced for adipogenic differentiation using adipogenic induction media for a period of seven days.

Result: A spindle shaped cell with accumulation of fat droplets was observed in differentiated cHFSCs. Differentiated cHFSCs were positively stained for Oil Red 'O' staining. Thus, this research proves that cHFSCs were multipotent cells which can be differentiated into many other type of cells such as adipocytes, chondrocytes, etc.
\end{abstract}

Key words: cHFSCs, CK15, Differentiation, Hair follicle, Stem cells.

\section{INTRODUCTION}

Stem cells are undifferentiated cells, defined by their ability at the single cell level to both self-renew and differentiate to produce mature progeny cells, including both non- renewing progenitors and terminally differentiated effector cells (Wagers and Weissman, 2004). The hair follicle was functioning as the "bone marrow of the skin" and continuously remodeled its cutaneous microenvironment including skin innervation and vasculature (Schmidt-Ullrich and Paus, 2005). Hair follicle stem cells (HFSC) were located at the bulge/isthmus region of the human hair follicles (Unna, 1876). The keratinocytes of the bulge region of mice hair follicles were quiescent and long-lived, multipotent (could give rise to all cell lineages of the hair follicle) and had high proliferative potential, thus fulfilling many of the traditional criteria for true stem cells (Lin and Andersen, 2008). Hair follicle stem cells were also characterized by expression of gene markers such as $\beta 1$ integrin,cytokeratin 15 , cytokeratin 19, $\alpha 6$ integrin, CD71, S100A, Caveolin1, CD200, PHLDA1, follistatin, frizzled homolog 1, nestin (neuron progenitor cell marker) and CD34 glycoprotein in human (Ohyama et al., 2006).

The hair follicle bulge cells were thought to differentiate into hair matrix keratinocytes, medulla, cortex, cuticle and basal sebocytes of the sebaceous glands and epidermis which could be used for wound healing (Lavker et al., 1993). Murine HFSCs differentiated into epidermis and called them as bipotent cells. They can also differentiate into neurons, glial cells, keratinocytes and smooth muscle cells in vitro in mice (Amoh et al., 2004). The HFSCs were implanted into gap region of a severed sciatic nerve. The HFSCs greatly enhanced the rate of nerve regeneration and the restoration of nerve function. The follicle stem cells transdifferentiated
Department of Veterinary Anatomy, Madras Veterinary College, Chennai-600 007, Tamil Nadu, India.

Corresponding Author: Sabiha Hayath Basha, Department of Veterinary Anatomy, Madras Veterinary College, Chennai-600 007, Tamil Nadu, India. Email: sabihahb@yahoo.com

How to cite this article: Hemavathi, N., Basha, S.H., Kumary, S.U. and Sivashankar, R. (2022). Adipogenic Differentiation of Canine Hair Follicle Stem Cells (cHFSCs). Indian Journal of Animal Research. DOI: 10.18805/IJAR.B-4735.

Submitted: 23-07-2021 Accepted: 05-01-2022 Online: 04-03-2022

largely into Schwann cells which were known to support neuron regrowth (Hoffman, 2006). Goat hair follicle stem cells (gHFSCs) were differentiated into osteocytes, chondrocytes and myocytes and differentiated osteocytes were confirmed for Von Kossa staining and expressed Osteocalcin. Differentiated chondrocytes were positive for Alcian blue staining and COL2A1 expression and myogenic induction confirmed by positive expression of gHFSCs for MyoG (He et al., 2016). Hence the present study was carried out to find the multipotent ability of canine hair follicle stem cells (cHFSCs) and differentiated as adipocytes.

\section{MATERIALS AND METHODS Ethical approval}

The research work has been carried out at the Department of Veterinary Anatomy, Madras Veterinary College, TANUVAS, Chennai during 2018-19 as per the approval of the Institutional ethical committee for stem cell research and therapy, Approval No.01/ICSCRT/2018 dated 23.11.2018 of the Chairman, ICSCRT, Madras Veterinary College, Chennai-07. 
Adipogenic Differentiation of Canine Hair Follicle Stem Cells (cHFSCs)

\section{Isolation and culture of cHFSCs}

Canine skin samples were collected and washed with $2 \%$ chlorhexidine for 3 minutes and washed with $70 \%$ isopropyl alcohol for a minute (Hibbard et al., 2002). Canine hair follicles were separated from skin by digesting them in Dispase-II enzyme (neutral protease, grade II; Roche; Cat. No. $04942078001 ; 2.4 \mathrm{U} / \mathrm{ml}$ in DMEM) at $4^{\circ} \mathrm{C}$ for overnight. In this method, to prepare single cell suspension, plucked canine hair follicles were placed into $0.25 \%$ trypsin-EDTA solution (SIGMA®; Cat. No. T4049) for digestion at $37^{\circ} \mathrm{C}$ in $\mathrm{CO}_{2}$ incubator for 15-20 minutes. Trypsin-EDTA was neutralized by adding serum and the digested bulge follicular cells were dissociated into single cell by gentle pipetting repeatedly and cultured in growth medium containing William's E medium (Sigma- Aldrich; W0397) or DMEM supplemented with $10 \%$ fetal bovine serum(FBS) (Gibco®; Cat. No. 10082-147) and $10 \mathrm{ng} / \mathrm{ml}$ of epidermal growth factor (EGF) (Thermofisher;PHG0311), $5 \mathrm{ng} / \mathrm{ml}$ of insulin growth factor (IGF-I; Cat. No. I8779) two per cent ABAM (ABAM) (Gibco®; Cat.No.15240-062) and one per cent amphotericin B (Sigma- Aldrich;Cat. No. A2942) in a humidified atmosphere at $37^{\circ} \mathrm{C}$ with 5 per cent $\mathrm{CO}_{2}$. Culture plates were coated with collagen coating solution (CELL; Cat. No. 12550) prior to seeding the cells. Cultures of cHFSCs were maintained upto passage 2 (P2) level for differentiation.

Cell proliferation(CD) and population doubling time (PDT) were calculated by following $C D=\ln (\mathrm{Nf} / \mathrm{Ni}) / \mathrm{In} 2(\mathrm{Nf}-$ final cell number; $\mathrm{Ni}$ - initial cell number; In- natural logarithm)(Spencer et al., 2012); PDT=1/r $\left(r=\log N_{2}-\log \right.$ $N_{1} / t_{2}-t_{1} ; N_{2}$ - total cells harvested at the hour of $t_{2} . N_{2}-$ total cells harvested at the hour of $t_{1}$ ) (Hilmi et al., 2013).

\section{Immunocytochemistry}

Cultured cells were fixed with $4 \%$ paraformaldehyde and permeabilized with $0.1 \%$ Triton $\mathrm{X}-100$ for 5 minutes and incubated with $8 \%$ blocking buffer for 25 minutes. Fixed cells were incubated with $250 \mu \mathrm{l}$ of primary antibody AntiCytokeratin 15 (CK15)(Cat. No. ab80522) $(5 \mu \mathrm{g} / \mathrm{ml})$ at $4^{\circ} \mathrm{C}$ for overnight and washed further $250 \mu$ of secondary antibody (Goat Anti-mouse IgG FITC conjugated) of 1:1000 dilutions was added and incubated for one hour and counterstained with DAPI. Washing with DPBS was followed after every step of incubation.

\section{Viability assessment}

Growth media was removed from cHFSCs culture plates and washed with DPBS. Cells were fixed with methanol for 5 minutes in room temperature. Fixative was removed and cells were washed with distilled water. Acridine orange stock solution was prepared by dissolving $1 \mathrm{~g}$ in $1000 \mathrm{ml}$ of Dulbecco's phosphate buffered saline (DPBS). For working solution, Acridine orange stock solution $(5 \mathrm{ml})$ was mixed with $20 \mathrm{ml}$ of $0.1 \mathrm{M}$ Citric acid and $1.3 \mathrm{ml}$ of $0.3 \mathrm{M}$ Sodium heptahydrate. Working solution was poured over the cell colonies and incubated for five minutes in room temperature. Culture plates were washed and observed under $530 \mathrm{~nm}$ filter for green fluorescence and $600 \mathrm{~nm}$ for red fluorescence.

\section{Adipogenic differentiation and staining}

cHFSCs of passage 2 (P2) with $70 \%$ confluency were incubated with adipogenic differentiation medium (Stem Pro $^{\mathrm{TM}}$ Adipogenesis Differentiation Kit; Gibco ${ }^{\mathrm{TM}}$; A1007001) for 7 days in $\mathrm{CO}_{2}$ in cubator. Adipogenic differentiation medium was changed for every two days. At the end of 7 days of induction, cells were fixed with $4 \%$ paraformaldehyde for 10 minutes in room temperature and washed with DPBS twice. Cells were stained with 0.3 per cent of Oil Red 'O' (Sigma $\AA$; 01391) for 50 minutes in room temperature to detect the lipid droplets in the cell cytoplasm.

\section{RESULTS AND DISCUSSION}

In primary culture of canine hair follicles bulge, round cells with clear cytoplasm was observed. On second day most of the cells were shown plastic adherence. The adhered cHFSCs showed small cytoplasmic processes surrounding round to oval nucleus with two or more nucleoli. The colonies became larger and united together forming a monolayer of cHFSC in the culture plate. Most of the colonies looked like cobblestone on appearance in the seventh day (Fig 1). Then cHFSCs were subjected to passage 1 (P1) (Fig 2) and passage 2 (P2) (Fig 3). The CD at primary culture was found

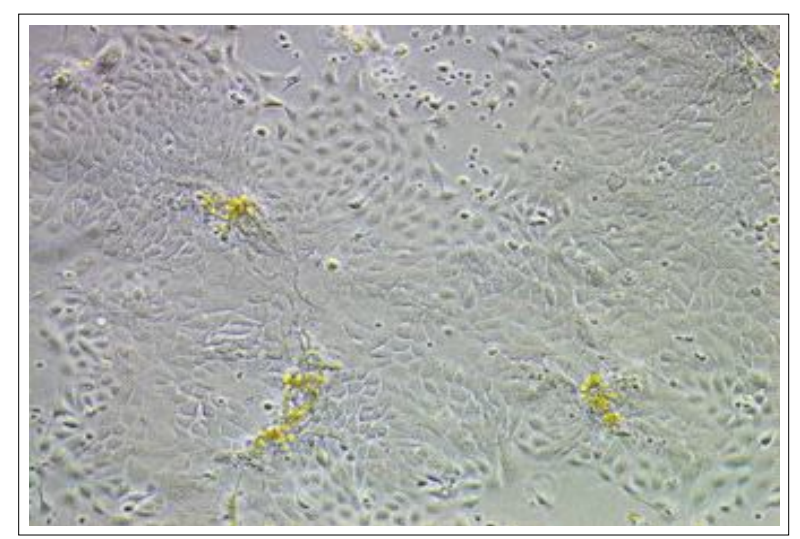

Fig 1: Photomicrograph of cHFSCs showing cobble stone morphology with 70-80 per cent confluency on seventh day of culture $(\times 200)$.

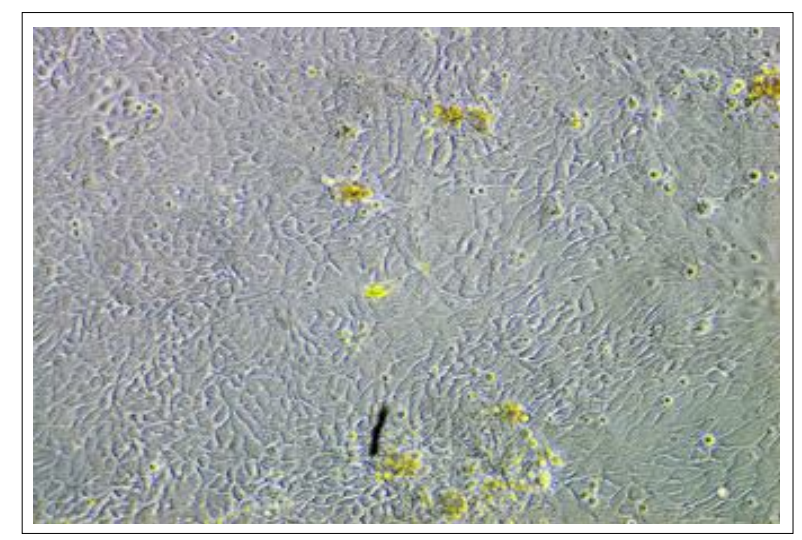

Fig 2: Photomicrograph of cHFSCs in P1 $(\times 200)$. 
Adipogenic Differentiation of Canine Hair Follicle Stem Cells (cHFSCs)

to be $0.11 \pm 0.21 \times 10^{6}$ which remained the same to $0.11 \pm 0.24 \times 10^{6}$ at $P 1$. The $C D$ at $P 2$ was higher than $P 1$ and measured about $0.49 \pm 0.10 \times 10^{6}$. The PDT of cHFSCs in primary culture was observed as $4.36 \pm 0.02$ hours in collagen coated culture plates. The PDT slightly increased in P1 as $4.38 \pm 0.02$ hours than primary culture. The PDT of P2 was found to be $4.29 \pm 0.06$ hours which was lesser than P1 culture.

\section{Immunocytochemistry}

The cultured cells of the hair follicle were immunostained with Anti-Cytokeratin 15 and DAPI on day seven of post incubation. The positive stem cells were confirmed by observing intra cytoplasmic green fluorescence and intra nuclear blue fluorescence (Fig 4).

\section{Acridine orange staining}

The viability of cells during culture was assessed by staining the primary cultured cells and P1 cells with Acridine orange. Viable cells were observed with emission of green fluorescence. The cells which were not viable appeared to emit light orange to red fluorescence in nucleus and yellow fluorescence cytoplasm. In primary culture, about 70 per cent of cHFSCs emitted green fluorescence (Fig 5 ).

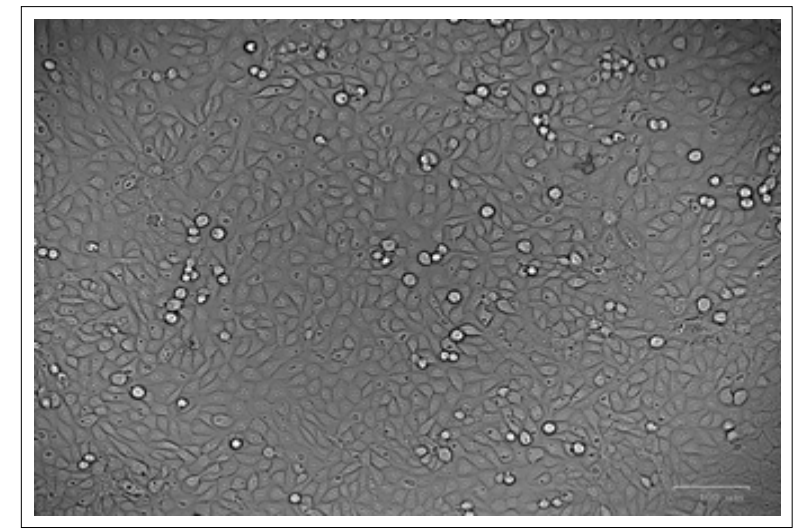

Fig 3: Photomicrograph of cHFSCs in P2 (100 $\mu \mathrm{m})$.

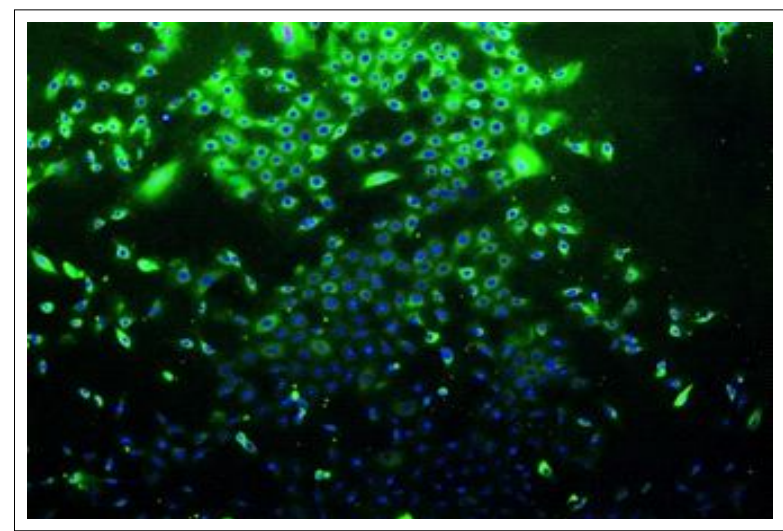

Fig 4: Photomicrograph of cHFSCs culture showing positive expression for Cytokeratin 15 (CK15) surface marker with DAPI $(\times 200)$.

\section{Adipogenic differentiated cHFSCs}

After incubation of cHFSCs in adipogenic media, on the second day of induction, the cell morphology changed from cobblestone appearance to long spindle shape cells with long cytoplasmic process (Fig 6). On day three, fat droplets were observed in the cytoplasm of differentiated cells and became rounded in appearance. On day seven of post induction with adipogenic media, cytoplasm of cells contained clusters of fat droplets (Fig 7) which were positive to Oil red ' $O$ ' staining (Fig 8).

In this study, cultured cHFSCs gave rise to macroscopic colonies within six days after initial seeding and colonies were homogenously made up of small flat cells near the periphery and the interior of the colony was stratified and consisted of large flat cells as described by Barrandon and Greens in human in 1987. Cells were closely aligned and gave cobblestone morphology which was similar to the results of goat and human hair follicle stem cell cultures respectively (Zhang et al., 2006; Hilmi et al., 2013).

In the present study, only flat keratinocyte type of cells were isolated from canine hair follicles whereas de Castro reported that mixed population of cells, mostly round and

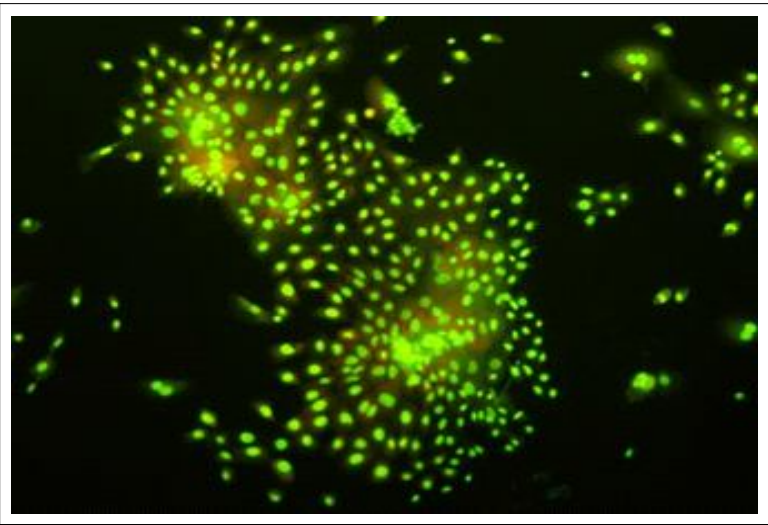

Fig 5: Photomicrograph of cHFSCs showing 90 per cent viable cells emitting green fluorescence-Acridine orange staining $(\times 100)$.

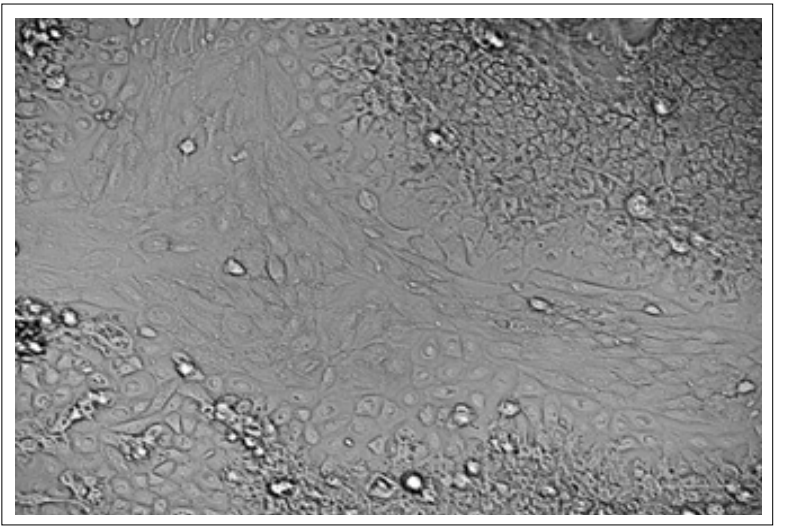

Fig 6: Photomicrograph of cHFSCs showing in vitro adipogenic differentiation: day-2 morphology of cHFSCs changed from flat cells into spindle shaped cells $(25 \mu \mathrm{m})$. 
Adipogenic Differentiation of Canine Hair Follicle Stem Cells (cHFSCs)

fusiform shaped cells were isolated from canine fetal and adult skin (de Castro et al., 2018).In the cell viability assay, the dead cells were observed as yellow to red in fluorescence and viable cells fluoresced green in colour as reported by Bank (1988). Sieber-Blum et al. (2004) reported that the initial doubling time of mouse HFSCs was approximately six hours and the PDT for human HFSCs cultured in uncoated plate with $\mathrm{CnT07}$ medium was $21.48 \pm 0.44$ hours and in KSFM coated plates, it was $30.73 \pm 0.75$ hours (Hilmi et al., 2013) which was reduced to $4.36 \pm 0.02$ hours in primary culture of cHFSCs cultured in collagen coated plates and PDT was decreased in P2 level and calculated as $4.29 \pm 0.06$ hours in the present study.

The expression of CK15 transcription factor and DAPI was demonstrated as intra cytoplasmic green fluorescence and intranuclear blue fluorescence respectively in cultured cHFSCs from 7-14 days of culture as in gHFSC (He et al., 2016) and in human HFSCs, positive expression was noticed along with CD200, CD34, K14, K15, CD271 and K1 markers (Hilmi et al.,2013; Inoue et al. 2009). Shen also performed immunofluorescence detection for K19 and $\beta 1$-integrin in human HFSCs (Shen et al., 2017). The hair follicle

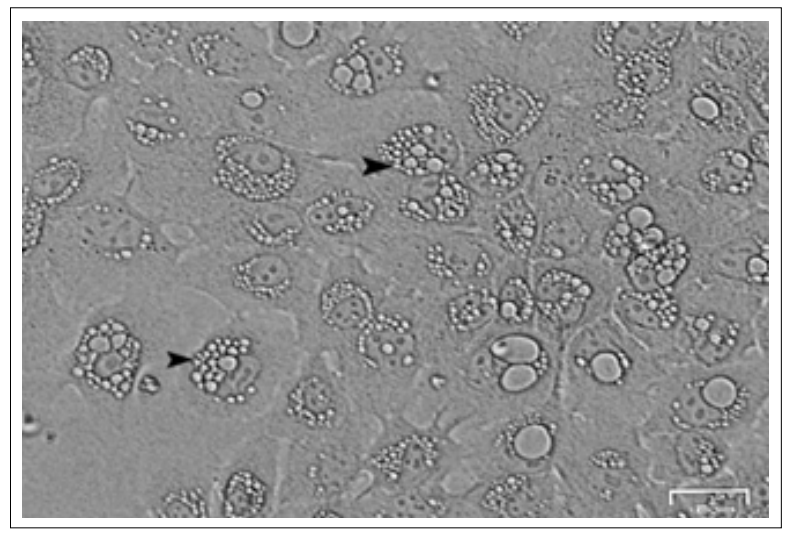

Fig 7: Photomicrograph of cHFSCs showing in vitro adipogenic differentiation (arrow heads) on seventh day of post induction $(25 \mu \mathrm{m})$.

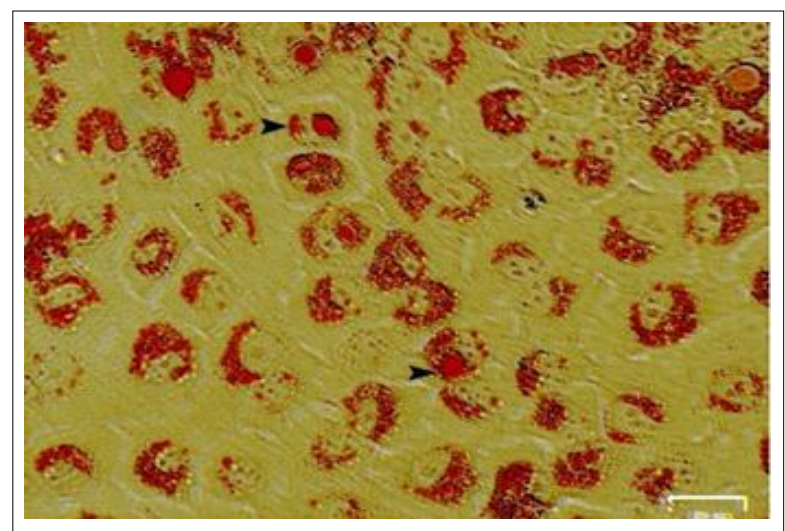

Fig 8: Photomicrograph of differentiated cHFSCs showing positive reaction to Oil red ' $O$ ' (arrow heads) after seven days of adipogenic induction $(25 \mu \mathrm{m})$. pluripotent nestin-driven GFP stem cells were positive for the stem cell marker CD34 but negative for CK15 and $\beta$-III tubulinin mice (Amoh et al., 2005).

Stem cells from dermal papilla of human hair follicles exhibited mesenchymal stem cell (MSC) immunophenotype and differentiated into all mesenchymal lineages and they were termed as human hair follicle derived mesenchymal stem cells (hHF-MSCs) (Liu et al., 2010). Adipogenesis property had decreased significantly in over time (Bajpai et al. 2012) Interestingly, loss of adipogenic differentiation potential was prevented by the adhesion of human Bone marrow mesenchymal stem cells to denatured type I collagen, suggesting a critical role of extracellular matrix in the maintenance of stem cell multipotency (Mauney et al., 2005). Adipogenic differentiation of canine hair follicle bulge stem cells has not been reported yet. In-vitro adipogenesis from cHFSCs was evident by aggregation of lipid rich droplets with cluster of differentiated cells stained positive by Oil red ' $O$ ' cytochemical staining after seven days of induction period. Review of earlier reports have evinced that the adipogenic differentiation of HFSCs from canine bulge cells was not proved in any species so far. Further validation of adipogenic differentiation of cHFSCs through qPCR is needed to confirm the differentiation property which was not performed in this study.

\section{ACKNOWLEDGEMENT}

We thank the Dean, Madras Veterinary College (MVC), TANUVAS, Chennai, for providing necessary funding to carry out the research. We acknowledge the Department of Surgery and Radiology, MVC, for providing skin samples and Centre for Stem Cell Research and Regenerative Medicine, MVC, for the lab facilities and guidance.

\section{Conflict of interest: None.}

\section{REFERENCES}

Amoh, Y., Li, L., Yang, M., Moossa, A.R., Katsuoka, K., Penman, S., Hoffman, R.M. (2004). Nascent blood vessels in the skin arise from nestin-expressing hair follicle cells. Proceedings of the National Academy of Sciences of the United States of America. 101(36): 13291-13295.

Amoh,Y., Li, L., Campillo, R., Kawahara, K., Katsuoka, K., Penman, S., Hoffman, R.M. (2005). Implanted hair follicle stem cells form Schwann cells that support repair of severed peripheral nerves. Proceedings of the National Academy of Sciences of the United States of America. 102: 1773417738.

Bajpai, V.K., Mistriotis, P., Andreadis, S.T., (2012). Clonal multipotency and effect of long-term in vitro expansion on differentiation potential of human hair follicle derived mesenchymal stem cells. Stem Cell Research. 8(1): 74-84.

Bank, H.L. (1988). Rapid assessment of islets viability with acridine orange and propidium iodide. In Vitro Cellular and Developmental Biology. 24(4): 266-273.

Barrandon, Y. and Greens, H. (1987). Three clonal types of keratinocyte with different capacities for multiplication. Proceedings of the National Academy of Sciences of the United States of America. 84(8): 2302-2306. 
Adipogenic Differentiation of Canine Hair Follicle Stem Cells (cHFSCs)

de Castro, R.V., Tavares, M.R., Bressan, F.F., Pieri, N.C., Hill, A.B.T., Souza, A.F., da R.N Cruz, N., Martins, D.S., Ambrósio, C.E., Meirelles, F.V.,Garcia, J.M. (2018). In vitro identification of a stem cell population from canine hair follicle bulge region. Tissue Cell. 50: 43-50.

He, N., Dong, Z., Tao, L., Zhao, S., Bou, S., Liu, D. (2016). Isolation and characterization of hair follicle stem cells from Arbas Cashmere goat. Cytotechnology. 68: 2579-2588.

Hibbard, J.S., Mulberry, G.K., Brady, A.R. (2002). A clinical Study Comparing the skin antisepsis and safety of chlora Prep, $70 \%$ isopropyl alcohol and $2 \%$ aqueous chlorhexidine. Journal of Infusion Nursing. 25(4): 244-249.

Hilmi, A.B.M., Halim, A.S., Noor, N.M., Lim, C.K., Idris, Z., Pohchi, A., Asma, H., Wahab, S.F.A., Tiede, S., Paus, R. (2013). A simple culture method for epithelial stem cells derived from human hair follicle. Central European Journal of Biology. 8(5): 432-439.

Hoffman, R.M. (2006). The pluripotency of Hair follicle Stem Cells. Cell cycle. 5(3): 232-233.

Inoue, K., Aoi, N., Sato, T., Yamauchi, Y., Suga, H., Eto, H., Kato, H., Araki, J., Yoshimura, K. (2009). Differential expression of stem-cell-associated markers in human hair follicle epithelial cells. Laboratory Investigation. 89(8): 844.

Lavker, R.M., Miller, S., Wilson, C., Cotsarelis, G., Wei, Z.G., Yang, J.S., Sun, T.T. (1993). Hair follicle stem cells: Their location, role in hair cycle and involvement in skin tumor formation. The Journal of Investigative Dermatology. 101(1): S16-S26.

Lin, K.K. and Andersen, B. (2008). Have Hair follicle Stem Cells Shed Their Tranquil Image? Cell Stem Cell. 3: 581-582.

Liu J.Y., Peng, H.F., Gopinath. S., Tian, J. Andreadis, S.T. (2010). Derivation of functional smooth muscle cells from multipotent human hair follicle mesenchymal stem cells. Tissue Eng Part A. 16: 2553-2564.

Mauney, J.R., Volloch, V., Kaplan, D.L., 2005. Matrix-mediated retention of adipogenic differentiation potential by human adult bone marrow-derived mesenchymal stem cells during ex vivo expansion. Biomaterials. 26(31): 61676175 .
Ohyama, M., Terunuma, A., Tock, C.L., Radonovich, M.F., PiseMasison, C.A., Hopping, S.B., Brady, J.N., Udey, M.C., Vogel, J.C. (2006). Characterization and isolation of stem cell-enriched human hair follicle bulge cells. Journal of Clinical Investigation. 116: 249-260.

Schmidt-Ullrich, R., Paus, R. (2005) Molecular principles of hair follicle induction and morphogenesis. Bioessays. 27(3): 247-26.

Shen, Q., Yu, W., Fang, Y., Yao, M., Yang, P. (2017). Beta-catenin can induce hair follicle stem cell differentiation into transitamplifying cells through c-myc activation. Tissue Cell. 49(1): 28-34.

Sieber-Blum, M., Grim, M., Hu, Y.F. and Szeder, V. (2004). Pluripotent neural crest stem cells in the adult hair follicle. Dev. Dynam. 231(2): 258-269.

Spencer, N.D., Chun, R., Vidal, M.A., Gimble, J.M. and Lopez, M.J. (2012). In vitro expansion and differentiation of fresh and revitalized adult canine bone marrow-derived and adipose tissue-derived stromal cells. Vet. J. 191(2): 231-239.

Unna, P.G. (1876). Histology and Development of Epidermis. Inaugural-Dissertation.

Wagers, A.J. and Weissman, I.L. (2004). Plasticity of adult stem cells. Cell. 116(5): 639-648.

Zhang, X., Wang, Y., Gao, Y., Liu, X., Bai, T., Li, M., Li, L., Chi, G., Xu, H., Liu, F., Liu, J.Y., 2013. Maintenance of high proliferation and multipotent potential of human hair follicle-derived mesenchymal stem cells by growth factors. International Journal of Molecular Medicine. 31(4): 913-921.

Zhang, Y., Xiang, M., Wang, Y., Yan, J., Zeng, Y., Yu, J., Yang, T. (2006). Bulge cells of human hair follicles: segregation, cultivation and properties. Colloids and Surfaces B: Biointerfaces. 47(1): 50-56. 